\title{
Design and Development of an Augmented Reality Robotic System for Large Tumor Ablation
}

\author{
Liangjing Yang ${ }^{1}$, Chee-Kong Chui ${ }^{1}$, Stephen Chang ${ }^{2}$ \\ ${ }^{1}$ Department of Mechanical Engineering, National University of Singapore, Singapore \\ ${ }^{2}$ Department of Surgery, National University Hospital, Singapore
}

\begin{abstract}
An augmented reality based robotic system is proposed for treatment of large liver tumor using multiple overlapping ablations technique. Radiofrequency ablation is a non-resection interventional procedure that minimizes invasiveness and reduces trauma. A single ablation is however limited by the ablation volume coverage. In treatment of tumor with diameter that could be $150 \mathrm{~mm}$ or more, a single needle insertion will not create sufficient ablation coverage to destroy the entire tumor. As such, multiple insertions are required. The precision and consistency associated with robot assisted surgery coupled with direct augmented reality projection system can better facilitate such treatment. The projection system is equipped with manipulator mechanism and robotic needle insertion arm for multiple insertions and needle deployment. The prototype augmented reality surgical system was evaluated on its abilities in ablating large tumor. Virtual models of the rectangular robot for projector manipulation and robotic needle insertion device were also used to assess the effectiveness of the proposed AR design.
\end{abstract}

Index Terms- augmented reality, image overlay, radiofrequency ablation, liver surgery, medical robotics

\section{INTRODUCTION}

Recently, there have been enormous efforts in the field of Augmented Reality (AR) and robotics for surgical applications [1-5]. AR usually involves the integration of virtual realistic model into real time view of the scene [3]. With the advancement in computer-based medicine, accurate diagnosis and precise pre-operative plans are available [6]. However, effective and consistent intra-operative execution remains a challenge. Surgeons are constantly faced with operation conditions that put them to extreme visual and dexterous constraints. The introduction of augmented reality creates a real time link between virtually constructed preoperative models to an intra-operative environment. This complements the visual capability of the surgeons. Coupled with robot assisted surgical system, operation can be executed with more consistency according to the surgical plan.

Radiofrequency (RF) needle ablation is a treatment technique that denaturizes tumor with heat created from ionic agitation generated by a needle electrode [7]. This form of treatment is a

Manuscript submitted May 6, 2008

E-mail: mpeyl@nus.edu.sg.mpecck@nus.edu.sg good alternative to liver resection. While resection of tumor surgically is by far the best option [8] for liver tumor, only $20 \%$ of liver cancer patients are suitable for open surgery [9]. Among the non-resectional procedures [7], RF ablation appears to be the most accepted treatment for liver tumors in terms of safety, ease of procedure and consistency $[10,11]$.

However, complete annihilation of the tumor is dependent on the ablation volume coverage. In treatment of large tumor with diameter that could be as large as $150 \mathrm{~mm}$, a single RF application is insufficient to destroy the entire tumor. As such, multiple needle deployments and insertions are required. There have been many research efforts on treatment strategies using multiple overlapping ablations [12-15]. Most of these researches revolve about planning and optimizing the ablation coverage to maximize effectiveness of the treatment. Although development significantly improves the effectiveness of pre-operative plans, achieving consistency and precision in intra-operative execution is still non-trivial. The technique of multiple needle insertions for overlapping ablation is difficult to perform manually. Surgeons are often deprived of visual information or have to rely highly on non-intuitive image guidance in executing the preplanned ablation model. Performing such operation manually will also be subjected to uncertainties and inconsistent outcomes [13]. We proposed systematic multiple ablations through robotic procedure guided by an AR image overlay for large tumor treatment using RF ablation.

This paper focused on the design and development of an AR based robotic system for multiple needle insertion in treating large tumor. The projector based AR system also includes a robotic system equipped with needle deployment and insertion manipulator.

\section{DESIGN AND DEVELOPMENT}

The construction of the AR surgical system involves designing the mechanical architecture of the system and the development of AR features. Apart from design consideration such as safety and sterilizability, there exist other design specifications unique to an AR based robotic surgical system. The design needs to be conceptualized based on the geometric requirements specified by the system kinematic model. During design conceptualization, specific application mechanism is developed concurrently. The virtual models of the generated 
design concepts are constructed using Computer Aided Design (CAD) software for engineering visualization and subsequent development. Actual physical prototype is implemented to test the performance and robustness of the design.

\subsection{Design considerations}

Safety is an important design consideration. However, safety guidelines for industrial robot are not always applicable to surgical robot. It is a requirement for industrial robot to be isolated from human operator when it is powered. Such practice is however difficult to implement in a surgical environment where both human operators and subjects cannot be excluded from the machine's workspace [16]. Therefore, there should be a reliable mechanism to cut off the power during an emergency as a form of engineering risk control. In an event of failure, the system has to be detachable from surgical tools easily to discontinue unexpected motion or enable manual operation so as to minimize interruption to operation. A modular approach for designing the robotic system will serve to facilitate the incorporation of such mechanism.

In addition, design should facilitate sterilization. Components having physical contact with patient need to be sterile. Even areas without contact should be covered with sterile sheet. In view of this, the modular approach is a good strategy. It will accommodate sterilization of components especially the end effecter of the needle manipulator which may contact the patient directly during an operation. Such component should be able to undergo sterilization in facilities like autoclave. The electromechanical manipulation system of the projector and the projector could all be covered with the sterile sheet.

\subsection{Kinematic model}

Interaction is an important concept in AR [2]. For effective link between virtual models and the real world spatially, some form of registration or calibration is essential. The pre-requisite to this procedure is the establishment of a kinematic model to the various interacting elements. The main mechanical components comprise of the projection system and the robotic needle insertion device. In the surgical system, components like the projector, camera, manipulator and RF needles are interactive elements. As such kinematic models are derived to represent these components spatially. In addition, the kinematic models describe the spatial interaction between components within the system thus providing a means for path planning and evaluation of the planned path.

The kinematic configuration of the projector manipulation robot is depicted in Fig. 1. In essence, it consists of a Cartesian configuration with two axles of control to position the projector in a plane at a given height. In addition, there are two axles of control to orientate the projector in pitch and yaw fashion.

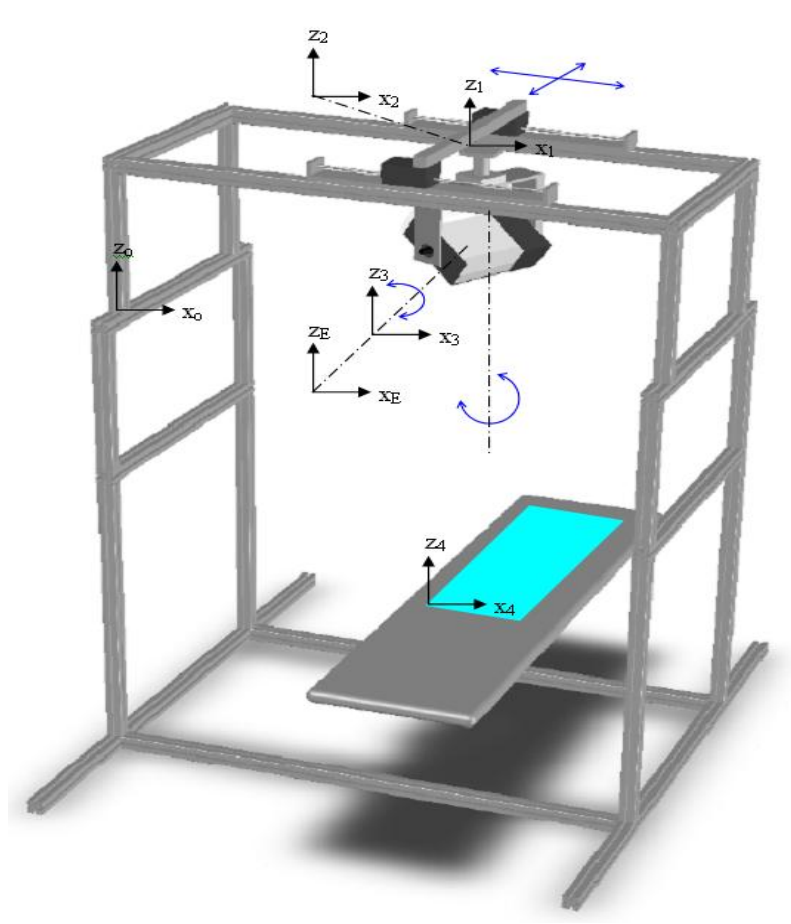

Fig. 1: Kinematic of projector manipulation robot. It is a rectangular robot

By multiplying the various transformation matrices, the homogenous matrix can be expressed in Equation 1.

$$
\begin{aligned}
& { }^{0} T_{1}=\left[\begin{array}{cccc}
1 & 0 & 0 & x_{1} \\
0 & 1 & 0 & y_{1} \\
0 & 0 & 1 & d_{1} \\
0 & 0 & 0 & 1
\end{array}\right] \quad{ }^{1} T_{2}=\left[\begin{array}{cccc}
c_{1} & -s_{1} & 0 & 0 \\
s_{1} & c_{1} & 0 & 0 \\
0 & 0 & 1 & 0 \\
0 & 0 & 0 & 1
\end{array}\right] \\
& { }^{2} T_{3}=\left[\begin{array}{cccc}
1 & 0 & 0 & 0 \\
0 & 1 & 0 & 0 \\
0 & 0 & 1 & d_{2} \\
0 & 0 & 0 & 1
\end{array}\right] \quad{ }^{3} T_{E}=\left[\begin{array}{cccc}
1 & 0 & 0 & 0 \\
0 & c_{2} & -s_{2} & 0 \\
0 & s_{2} & c_{2} & 0 \\
0 & 0 & 0 & 1
\end{array}\right] \\
& { }^{0} T_{E}=\left[\begin{array}{cccc}
c_{1} & -s_{1} c_{2} & s_{1} s_{2} & x_{1} \\
0 & c_{1} c_{2} & c_{1} s_{2} & y_{1} \\
0 & s_{2} & c_{2} & d_{2} \\
0 & 0 & 0 & 1
\end{array}\right]
\end{aligned}
$$

The design of the robotic needle insertion device is dependent on the kinematics configuration assuming a multi-body dynamic model. Schematics representing the kinematic configuration of the robotic system are analyzed. Frames and link parameters are assigned based on the Denavit-Hartenberg convention for the derivation of the governing kinematics represented by transformation matrix. Fig. 2 is a schematic overview of the kinematic analysis. While there is a need to deliver the treatment within a reasonable speed, the precision must not be compromised. The design concept where a sub-manipulator system is installed at the distal end of the main manipulator is implemented. This design concept allows fast motion to be 
carried out by the main manipulator and fine positioning executed by the sub-manipulator, hence achieving both swiftness and precision. A cylindrical manipulator configuration was proposed for the design of the main manipulator system. This main manipulator system will accommodate the proposed sub-manipulator system as illustrated in the figure. The sub-manipulator will consist of an $\mathrm{x}-\mathrm{y}$ translation mechanism and a flexible arm for maneuvering the end effecter over the contour of the liver organ.

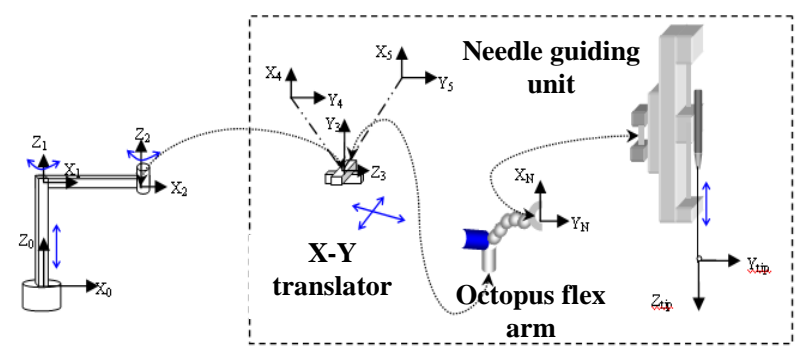

Main Manipulator System Sub-manipulator System

Fig. 2: Kinematic of serial robotic manipulator insertion device

The governing kinematics of the design can be represented by transformation matrix as shown in Equation 2.

$$
{ }^{0} T_{\text {tip }}={ }^{0} T_{5}{ }^{5} T_{N}{ }^{N} T_{\text {tip }}
$$

${ }^{0} T_{5}$ describes the spatial relationship between the globally fixed Frame 0 and the $x-y$ translational stage. It is expressed as follow

$$
{ }^{0} T_{5}=\left[\begin{array}{cccc}
0 & s_{13} & c_{13} & t_{y} c_{13}+t_{x} s_{13}+l_{2} c_{1} \\
0 & -c_{13} & s_{13} & t_{y} s_{13}-t_{x} c_{13}+l_{2} s_{1} \\
1 & 0 & 0 & l_{1} \\
0 & 0 & 0 & 1
\end{array}\right],
$$

where $s_{j}=\sin \left(q_{i}\right), c_{i j}=\cos \left(q_{i}\right)$,

$$
\begin{aligned}
& s_{i j}=\sin \left(q_{i}+q_{j}\right), c_{i j}=\cos \left(q_{i}+q_{j}\right), \\
& s_{i j k}=\sin \left(q_{i}+q_{j}+q_{k}\right), c_{i j k}=\cos \left(q_{i}+q_{j}+q_{k}\right) .
\end{aligned}
$$

${ }^{5} \mathrm{~T}_{\mathrm{N}}$ is the transformation matrix from Frame 5 to Frame $N$, the multi-segmented grip. $N$ represents the number of segments. The current prototype uses an octopus flex-arm consisting of 22 segments. It can be expressed as follow

$$
{ }^{5} T_{N}=\left[\begin{array}{cc}
{ }^{5} R_{N} & \sum_{i=5}^{N}{ }^{5} R_{i}{ }^{i} P_{i+1} \\
0 & 1
\end{array}\right]
$$

where ${ }^{a} R_{b}$ is the rotational matrix from Frame $a$ to $b,{ }^{a} P_{b}$ is the translational matrix from Frame $a$ to $b$, and $N$ is the number of segments.

Similarly, ${ }^{\mathrm{N}} \mathrm{T}_{\text {tip }}$ represents the orientation and position of the assigned frame at the needle tip with respect to Frame N. This part of the manipulator consists of only one degree of freedom. It can be expressed as follow

$$
\text { A. }{ }^{5} T_{N}=\left[\begin{array}{cccc}
0 & 0 & -1 & -r \\
0 & 1 & 0 & d \\
1 & 0 & 0 & 0 \\
0 & 0 & 0 & 1
\end{array}\right],
$$

where $r$ is the displacement of insertion/withdrawal joint and $d$ is the offset from grip to insertion joint axis. Based on current prototype, $\mathrm{d}$ has a value of $62 \mathrm{~mm}$.

A conservative estimation of the workspace for RF ablation treatment sufficient to cover the entire liver will be a planar area of $180 \mathrm{~mm}$ by $180 \mathrm{~mm}$ assuming insertion trajectory to be vertical. Hence any goal point must be within the system workspace.

Three dimensional virtual models of the overhead rectangular robot and serial robotic manipulator are built on CAD software, SolidWorks to prepare for prototyping. Fig. 3 and 4 shows the virtual model of the projector manipulation robot and the robotic needle insertion device for design visualization and simulation of the kinematic interactions with the surgical environment. It is designed such that the needle insertion device poses minimal obstruction to the projected image as well as the surgeon.

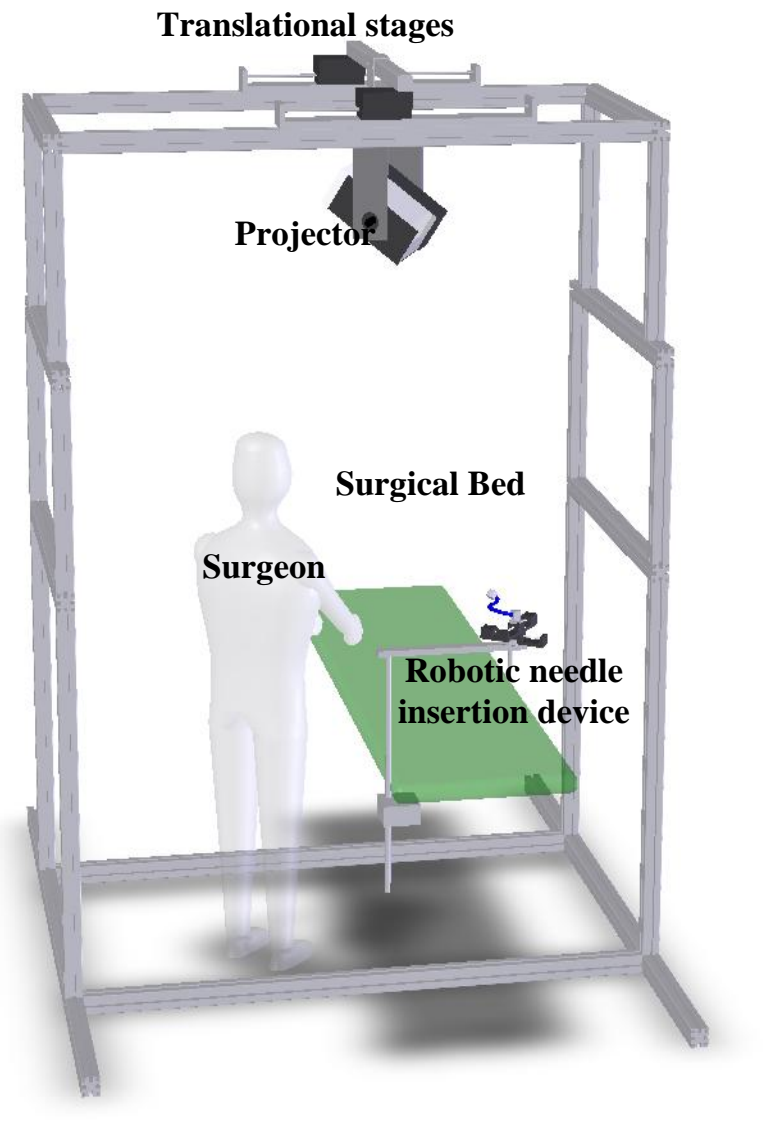

Fig. 3: Virtual model of AR robotic surgical system 


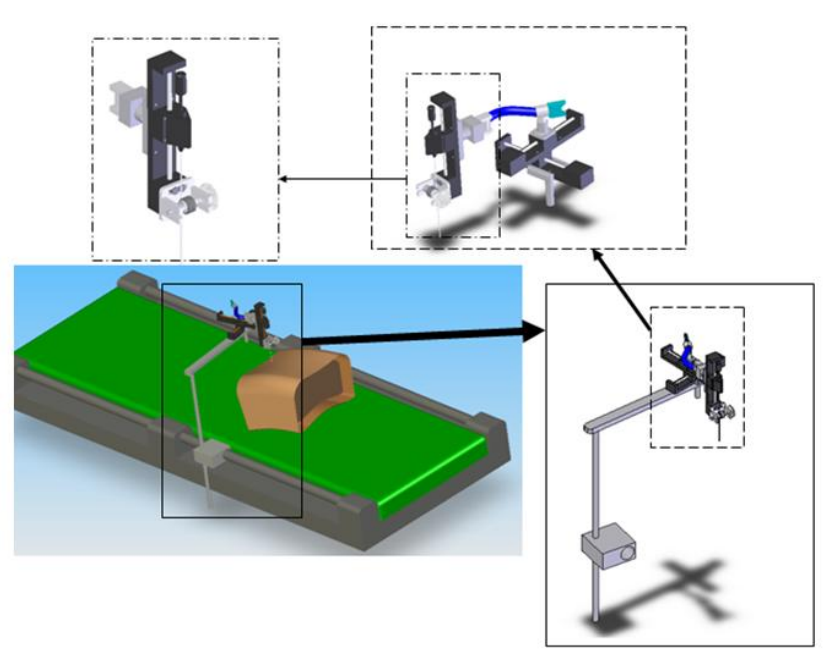

Fig. 4: Virtual model of robotic needle insertion device in surgical field

\subsection{System Components}

The overhead rectangular robotic projection system consists of aluminum profiles assembled structural frame and an adjustable projector housing. The upper frame can be dismantled from its base and mount onto ordinary workbench for development and testing of the AR system. Fig. 5 illustrates our current testing environment. The serial robotic manipulator for needle insertion is integrated into the system as featured in Fig. 6. The structural frame was designed to be assembled and dismantled easily. This is to facilitate future work in animal studies where the system will be set up in surgical theatre. Motion control is implemented with ball-screw translational stages and motorized mechanism driven by fine resolution stepper motors. Apart from motion control, the electromechanical manipulation system provides geometrical awareness to the system for tracking of the kinematics of the components.

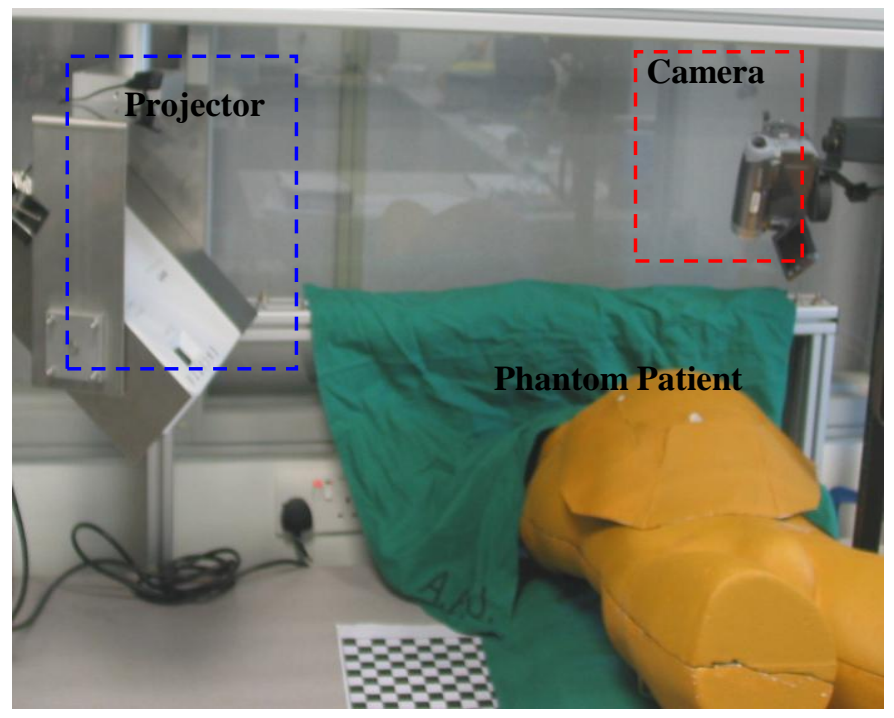

Fig. 5: Projection system testing workbench

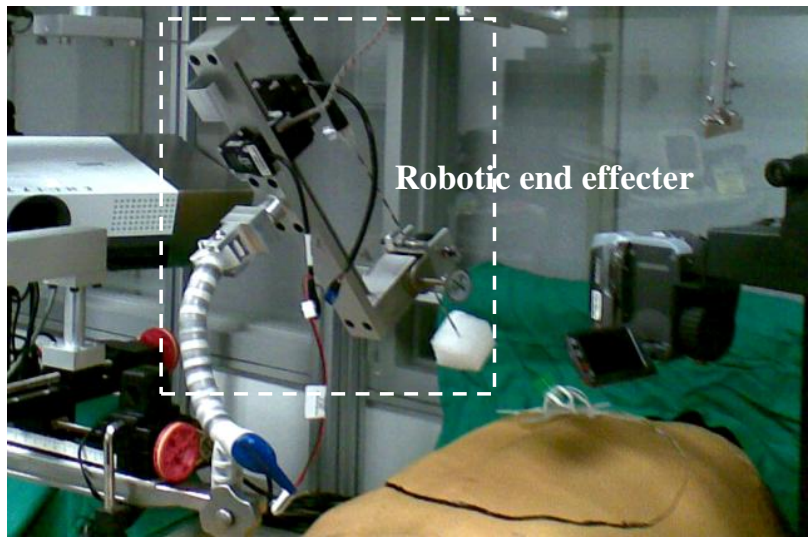

Fig. 6: Incorporation of robotic needle insertion into the projection system

Fig. 7 describes the prototype of the serial robotic manipulator for performing needle insertion. This prototype is a realization of the developed mechanical model described above. The robotic prototype consists of a 3 DOF manipulator system and a distal end sub-manipulator system of $48 \mathrm{DOF}$. The robotic system uses the design concept of sub-manipulator to deliver multiple insertions with consistency. The motorized sub-manipulator system is responsible for consistent and accurate deployment of the needle. Needle insertion is executed by a linear insertion actuator. This actuation system is guided by precise translation stage. The resolution of the linear translation is $0.25 \mathrm{~mm}$ and the system is capable of a maximum insertion speed of $4 \mathrm{~mm} / \mathrm{s}$. A needle guiding unit is attached to the insertion actuator to ensure linear trajectory during needle insertion. The needle guiding unit is a detachable module that stabilize linear trajectory of the needle shaft with elegant mechanical means. It rectifies any possible needle deflections by stabilizing the insertion with dual plane roller guide. This wheel also bears a knob that controls manual driven insertion. In event of system failure, the needle can be rapidly detached from the motor controlled grip via a quick release mechanism and resume operation using the manual knob. This targeting device consisting of a pair of laser pointers designed to create a visual marker on the surface or the projected image to indicate the point of insertion.

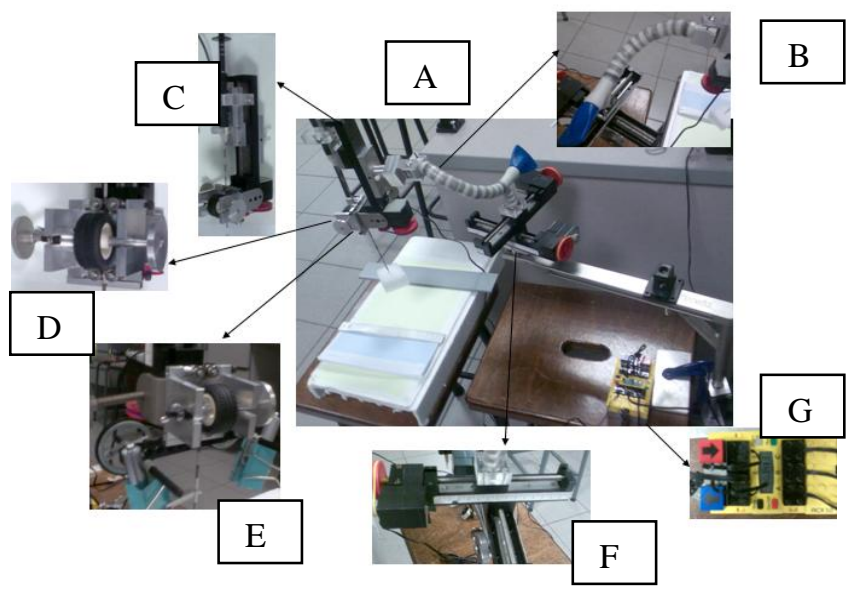

Fig. 7: (A)Overview of robotic system (B)Octopus Flex Arm (C)Insertion actuator (D)Needle guiding unit (E)Targeting device (F)Translation mechanism $(\mathrm{G}) \mathrm{RCX}$ controller 


\subsection{Operating Mechanism}

The complete ablation of large tumor can be achieved by a systematic multiple needle deployment that plot out the required volume of ablation coverage. Dodd et al [13] derived various overlapping ablation models based on computer analysis of RF ablation thermal injury. One of them is the cylindrical model. Though it was found to be less efficient, the model offers ease of control. Most importantly, the systematic nature of the model enables intuitive and flexible execution planning. Further development was carried out by Chen et al [14] in a clinical study of 110 patients to establish the protocol for RF ablation treatment in large liver tumor. The protocol was developed based on a regular prism and polyhedron model. The developed serial robotic manipulator is capable of executing a trajectory for the cylindrical model. The model consists of spherical ablation volumes with uniform lateral intervals between centers of ablation spheres. This systematic row of ablation zone can eventually be extended to an array of larger ablation coverage. Our proposed operation mechanism applied the concept of sub-manipulator system at the distal end of the main manipulator system to execute precise deployment of needle under intra-operative AR guidance so as to cover accurately the entire volume of tumor as shown in Fig. 8.

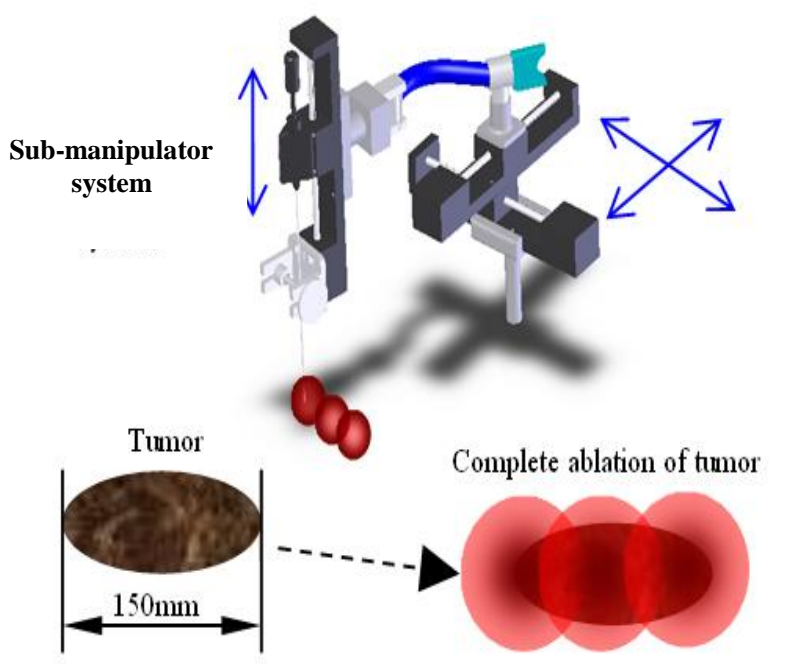

Fig. 8: Overlapping multiple ablations model with sub-maniplulator

The kinematics involves simple translation within the specific space constraint depending on the surgery. Hence a sub-manipulator system consisting of precise $x-y$ translation mechanism and flexible link is incorporated at the distal end for fine adjustment. Together with the high precision insertion actuator, the system is capable of positioning the needle at any point precisely and rapidly in three-dimensional free space.

Fig. 9 is the process flow of an effective procedure that combines the consistency of the robotic system and visualization aid through $\mathrm{AR}$.

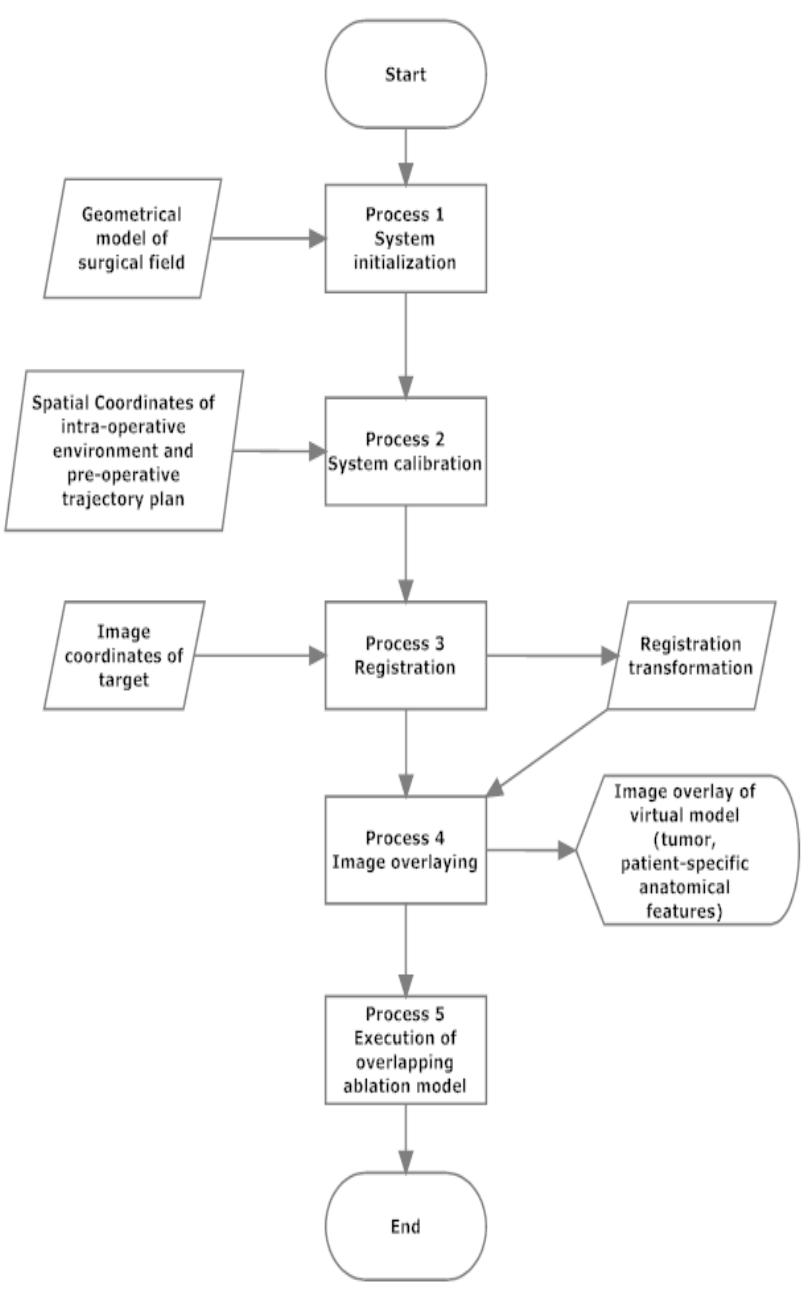

Fig. 9: Operation process flow of the AR robotic system

\section{Process 1: System initialization}

This includes assigning a global frame of reference based on the geometrical model of the surgical field. The aim is to establish spatial reference to the surgical system and enable registration of the pre-operative planning to the intra-operative environment subsequently.

\section{Process 2: System calibration}

In essence, the serial robotic manipulator for needle insertion has to be calibrated to the overhead rectangular robot for projector manipulation. This includes the derivation of a geometrical transformation describing the spatial relationship between the two sub-systems. This can be done by processing the kinematic models discussed previously with geometric parameters. The transformation matrix will subsequently enable the expression of kinematics of the components to be referenced in the same domain.

\section{Process 3: Registration}

Registration in the AR context refers to the creation of relation awareness between the real environment and its corresponding virtual representations [1]. The key difference between AR and VR lies in the fact that VR only involves virtual models existing in the virtual environment while AR complements the real world 
environment with virtual model. Hence virtual models need to be reference with respect to real world coordinates. This can be done by a few developed techniques each with different system requirements [17].

\section{Process 4: Image overlaying}

This process includes application of the registration transformation obtained from process 3 to manipulate the image projection such that the targets corresponds to the tumors and that the image data is matched accurately to its corresponding features. The image coordinate of the targets should be derived from pre-operative computer processed imaging and surgical plan.

\section{Process 5: Execution of overlapping ablation model}

Multiple needle insertion and deployment of needle will be done under intra-operative guidance of the AR projection.

\section{EXPERIMENT}

The camera-projector system involves solving the homography to achieve registration of patient pre-operative and intra-operative images. There is an application to non-planar projection. Fig. 10 is an example of direct projection of image data onto the phantom object. The serial robotic manipulator for needle insertion can target all sectors of the liver organ.

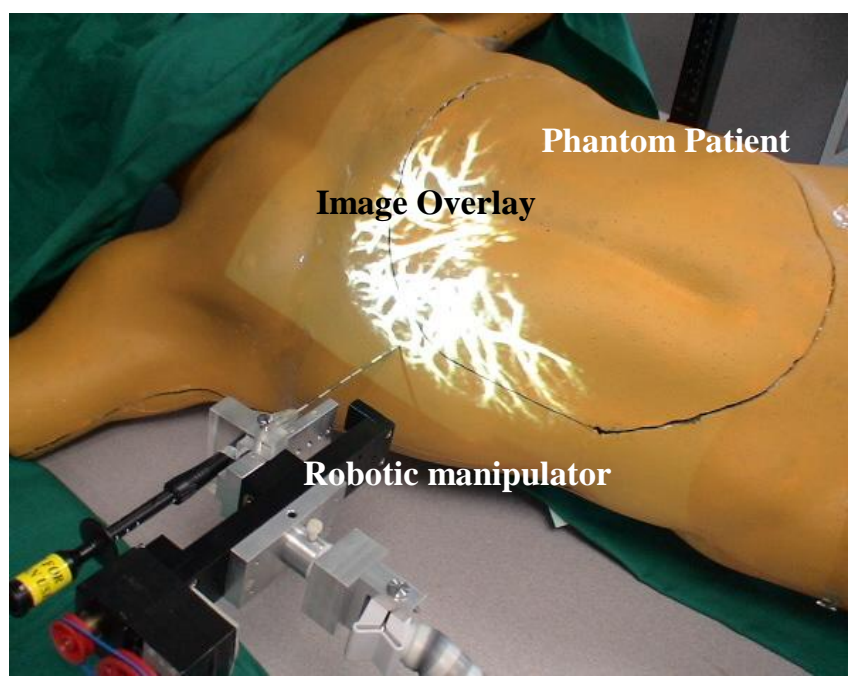

Fig. 10: Image overlay on phantom patient for AR guidance surgery

A series of tests were conducted to assess the performance of robotic needle insertion device. The reliability of the serial robotic manipulator is the key requirement for multiple overlapping ablations of our AR robotic system. An experiment test-bed was set up to carry out the experiments. Multi-layer phantom tissues made of agar were used. This tissue mimicking phantom model acts as the needle path medium to simulate realistic insertion strokes. Experiments are designed based on the established operation mechanism taking into account actual application and clinical factors. An illustration of the experiment set-up is shown in Fig. 11.

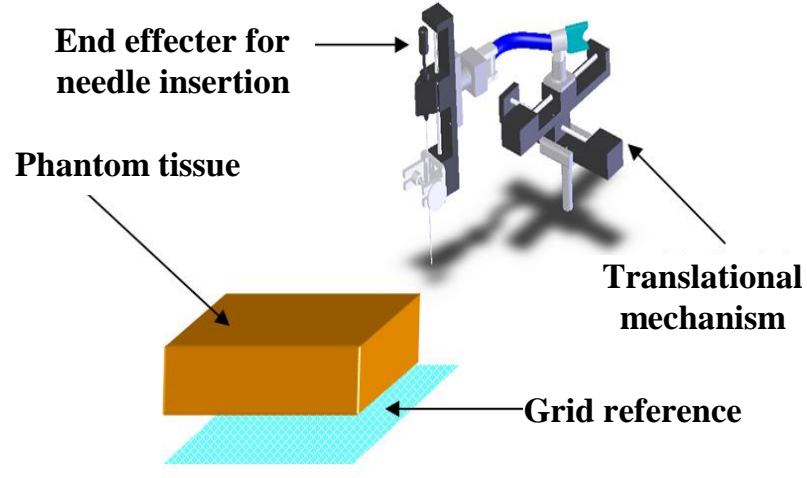

Fig. 11: Experimental set up with phantom tissue model made of agar.

The first experiment is to test the proposed needle guiding concept. Needle insertion and withdrawal was done on the same point on a phantom liver from an insertion height of $160 \mathrm{~mm}$. A reference grid was overlaid on the phantom specimen to register the positions of five punctures. These five insertions are intended to puncture the same point. Hence it is desired that the five punctures are as close as possible to the intended point. The desired point is marked by a visual marker produced by targeting LED. Deviation of punctures from this theoretical point will be registered as the error. This experiment attempts to test the system accuracy in insertion path directed at target. All the insertions were done using RITA StarBurst XL needle. This is a commercially available RF needle used for tumor ablation during hepatobiliary surgery. The results are tabulated in Table 1 .

A motion consistency test was designed to assess the precision of the executed ablation model. In this experiment, the manipulator will execute the assigned trajectory as in actual intra-operative situation. The actual path taken by the needle tip will be recorded. In this experiment, we adopted the cylindrical model to be the pre-operatively derived plan. In order to plot out ablation pattern of this model, the needle tip will have to be deployed in uniform intervals as discussed in previous section. As such the virtual markers to be augmented on the operative field will be in a linear configuration. The positioning of the end effecter by $x-y$ translation mechanism is tested. With the RF needle affixed, the serial robotic manipulator executes a cyclical series of sequential insertion, withdrawal and translation of the needle via a layer of phantom tissue resting on top of polystyrene with grid overlay. Fig 12 illustrates the execution path for the experiment. The deviation from the intended linear pattern is tabulated in Table 2 .

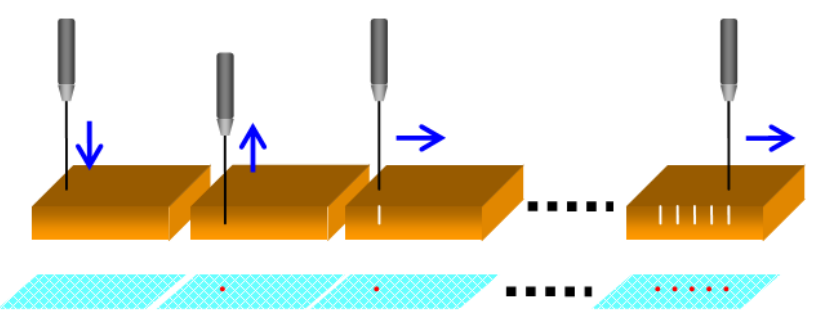

Fig. 12: Illustration of motion consistency test 


\section{RESULTS}

From Table 1 we see that the implementation of the needle guiding unit reduces the mean error by $0.21 \mathrm{~mm}$ which is $22.1 \%$. This suggests that the guiding unit effectively stabilize needle deflection during the insertion stroke. Therefore apart from its guiding function for manual insertion, the guiding unit is also important for accurate automated insertion. The performance of the system in this test is important as accurate targeting of a single target is the most important prerequisite for multiple needle insertion.

\section{TABLE 1: RESULT OF ACCURACY TEST}

\begin{tabular}{ccccc}
\hline \multicolumn{5}{c}{ Motorized without needle guiding unit } \\
\hline $\begin{array}{c}\text { Experimental } \\
\text { point }\end{array}$ & $\begin{array}{c}\mathrm{X} \\
(\mathrm{mm})\end{array}$ & $\begin{array}{c}\mathrm{Y} \\
(\mathrm{mm})\end{array}$ & $\begin{array}{c}\mathrm{r}^{2} \\
\left(\mathrm{~mm}^{2}\right)\end{array}$ & $\begin{array}{c}\mathrm{r} \\
(\mathrm{mm})\end{array}$ \\
1 & -0.5 & 1 & 1.25 & 1.12 \\
2 & -1 & 0.5 & 1.25 & 1.12 \\
3 & -0.5 & -1 & 1.25 & 1.12 \\
4 & -1 & -1 & 2 & 1.41 \\
5 & 0 & 0 & 0 & 0 \\
\hline \multicolumn{5}{c}{ Mean $\mathrm{r}=0.95$} \\
\hline Mxperimental & $\mathrm{x}$ & $\mathrm{Y}$ & $\mathrm{r}^{2}$ & $\mathrm{r}$ \\
point & $(\mathrm{mm})$ & $(\mathrm{mm})$ & $\left(\mathrm{mm}^{2}\right)$ & $(\mathrm{mm})$ \\
1 & 0.5 & 0.5 & 0.5 & 0.71 \\
2 & 0.5 & 0.5 & 0.5 & 0.71 \\
3 & -0.5 & -0.5 & 0.5 & 0.71 \\
4 & -0.5 & -0.5 & 0.5 & 0.71 \\
5 & -0.5 & -0.5 & 0.5 & 0.71 \\
\hline \multicolumn{5}{c}{}
\end{tabular}

Fortunately, this magnitude of error does not have significant clinical implication. According to the general practice for RF ablation treatment, a safety margin of $10 \mathrm{~mm}$ in excess to tumor boundary is usually adopted [18]. The performance test has shown that error of the developed robotic manipulator system is capable of achieving insertion and motion trajectory execution within an error of $1 \mathrm{~mm}$ for the intended paths. This error is safely kept within the $10 \mathrm{~mm}$ margin. The volumetric misalignment can be reflected by the amount of volume that fails to be covered in an actual insertion. Take the ablation spherical volume to be of diameter $40 \mathrm{~mm}$ which is the technically recommended standard for most RITA StarBurst XL needle. Mean error of insertion with insertion actuator and needle guiding unit is $0.71 \mathrm{~mm}$. By calculus derivation, the volumetric misalignment is approximately $891.8 \mathrm{~mm}^{3}$. Comparing with the entire ablation volume of $33510 \mathrm{~mm}^{3}$ it is an error of $2.66 \%$.

The result for the motion consistency test is also promising. The maximum deviation from the desire path is not more than $0.5 \mathrm{~mm}$. Fig. 13 illustrates the deviation of the actual ablation pattern from the intended path planned.

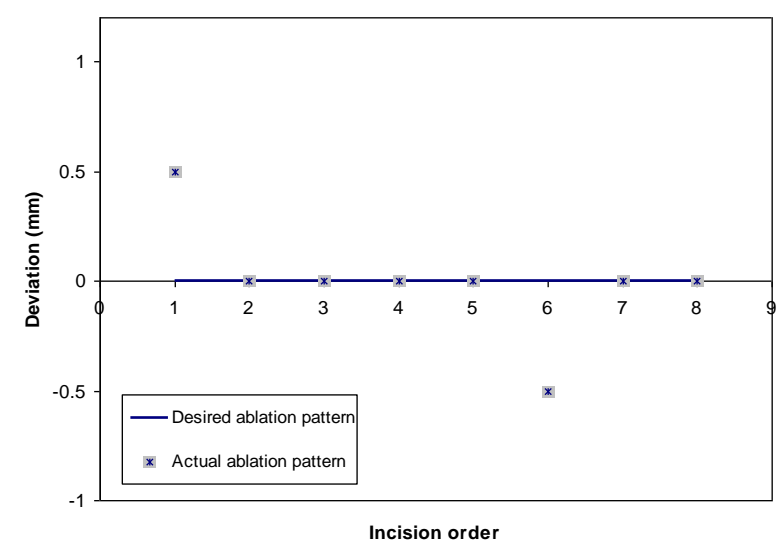

Fig. 13: Deviation of actual and desired path

With a resolution of $0.5 \mathrm{~mm}$, there is hardly any observable deviation except for a few occasions. The error in this experiment is very much due to needle deflection when it travels through the layers of agars. It has been explained in studies, that the unbalanced resistance load on needle tip during insertion resulted in needle deflection $[19,20]$.

\begin{tabular}{cc} 
TABLE 2: RESULT OF MOTION CONSISTENCY TEST \\
\hline Incision order & Deviation $(\mathrm{mm})$ \\
\hline 1 & 0.5 \\
2 & 0 \\
3 & 0 \\
4 & 0 \\
5 & 0 \\
6 & -0.5 \\
7 & 0 \\
8 & 0 \\
\hline & Mean $=0$ \\
\end{tabular}

\section{DISCUSSION AND CONCLUSION}

An AR robotic system for multiple needle insertion is proposed and developed to successfully treat large tumor. It is capable of reliable performance in term of accurate visualization, precise motion execution capabilities. The kinematics and mechanics of the manipulators for projector and needle insertion mechanism are derived from first principle and geometrical approach mathematically. Prototype is constructed and experiments have been performed with the prototype system. Data have supported the feasibility of the AR surgical setup which yields promising results in terms of accuracy and precision.

The developed serial robotic manipulator is designed based on a geometrically efficient kinematic model. The key advantage of the developed mechanism is its straightforward governing kinematics that lay a foundation for an intuitive operation path as compared to conventionally sophisticated articulating manipulator system. Generally, it would have been argued that articulated robots are less workspace intrusive and dexterous [21]. However through a problem specific approach, 
this study has shown that the proposed configuration can be more appropriate for multiple RF ablations, as well as displaying of image overlays on patient. The computation of the manipulator dynamics is less demanding because of the Cartesian configuration. In the case of the needle insertion serial robotic manipulator, the load sensor is not required for the dynamic control of the manipulator joints in contrast to the usual articulated manipulators.

The establishment of the manipulation mechanism also provides a platform for integration of existing research findings on soft tissue needle insertion with robotic devices. There have been a lot of research efforts directed toward understanding the needle-tissue interaction and modeling a realistic simulation of the interaction [22, 23]. This existing knowledge can be combined with the developed serial robotic manipulator mechanics for a more holistic model of robotic assisted needle insertion device.

Nevertheless, achieving accurate and realistic visual augmentation in the real-time surgical field remains challenging. The virtual anatomical models have been built from diagnostic imaging modules like MRI or CT. As seen in Fig. 14, the vasculature of the liver is displayed clearly to provide guidance in navigating surgical tools. We are developing effective algorithm for correction of geometric distortion characterized by high degree of elasticity transformation. To enhance the realism, radiometric compensation is also explored.

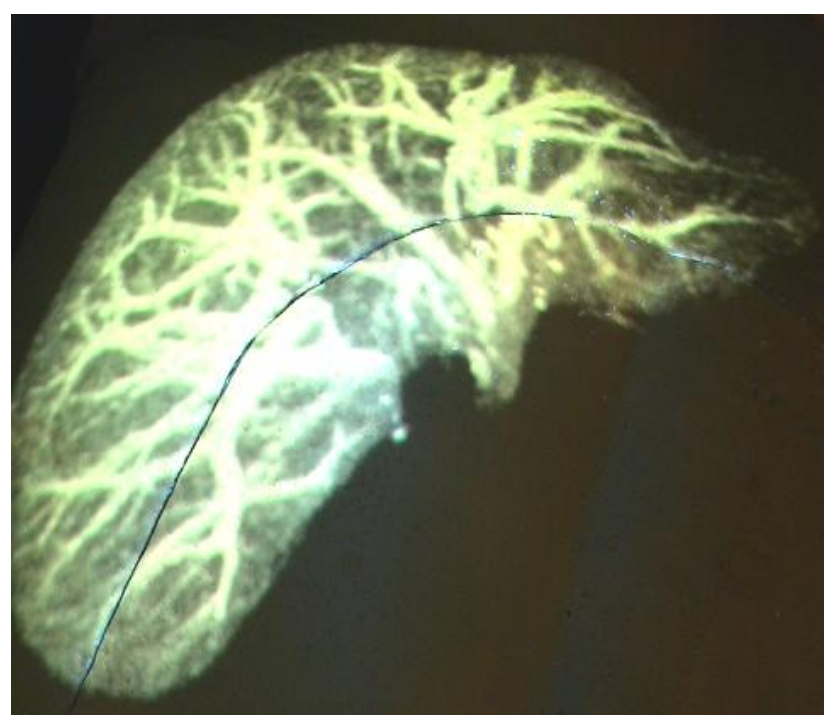

Fig. 14: Image overlay on the phantom displaying vessels of the liver organ.

\section{ACKNOWLEDGMENT}

This research is supported by a grant from National University of Singapore (R-265-000-270-112 and R-265-000-270-133).

\section{REFERENCES}

[1] M. Blackwell, C. Nikou, A. M. DiGioia, and T. Kanade, "An Image Overlay system for medical data visualization," Medical Image Analysis, vol. 4, pp. 67-72, 2000.
[2] J. Marescaux, L. Soler, and F. Rubino, "Augmented Reality for Surgery and Interventional Therapy," Operative Techniques in General Surgery, vol. 7, pp. 182-187, 2005.

[3] D. A. Wang, F. Bello, and A. Darzi, "Augmented reality provision in robotically assisted minimally invasive surgery," International Congress Series, vol. 1268, pp. 527-532, 2004.

[4] N. Abolhassani, R. Patel, and M. Moallem, "Experimental study of robotic needle insertion in soft tissue," International Congress Series, vol. 1268, pp. 797-802, 2004.

[5] M.-A. Janvier, L.-G. Durand, M.-H. R. Cardinal, I. Renaud, B. Chayer, P. Bigras, J. de Guise, G. Soulez, and G. Cloutier, "Performance evaluation of a medical robotic 3D-ultrasound imaging system," Medical Image Analysis, vol. 12, pp. 275-290, 2008.

[6] R. H. Taylor, P. Kazanzides, and F. David Dagan, "Medical Robotics and Computer-Integrated Interventional Medicine," in Biomedical Information Technology Burlington: Academic Press, 2008, pp. 393-416.

[7] S. Benoist and B. Nordlinger, "Radiofrequency ablation in liver tumours," Ann Oncol, vol. 15 Suppl 4, pp. iv313-7, 2004.

[8] B. Nordlinger, M. Guiguet, J. C. Vaillant, P. Balladur, K. Boudjema, P. Bachellier, and D. Jaeck, "Surgical resection of colorectal carcinoma metastases to the liver. A prognostic scoring system to improve case selection, based on 1568 patients. Association Francaise de Chirurgie," Cancer, vol. 77, pp. 1254-62, Apr 11996.

[9] "Primary liver cancer in Japan. Clinicopathologic features and results of surgical treatment. Liver Cancer Study Group of Japan," Ann Surg, vol. 211, pp. 277-87, Mar 1990.

[10] W. Y. Lau, T. W. Leung, S. C. Yu, and S. K. Ho, "Percutaneous local ablative therapy for hepatocellular carcinoma: a review and look into the future," Ann Surg, vol. 237, pp. 171-9, Feb 2003.

[11] J. P. McGhana and G. D. Dodd, 3rd, "Radiofrequency ablation of the liver: current status," AJR Am J Roentgenol, vol. 176, pp. 3-16, Jan 2001.

[12] T. F. Wood, D. M. Rose, M. Chung, D. P. Allegra, L. J. Foshag, and A. J. Bilchik, "Radiofrequency ablation of 231 unresectable hepatic tumors: indications, limitations, and complications," Ann Surg Oncol, vol. 7, pp. 593-600, Sep 2000.

[13] G. D. Dodd, 3rd, M. S. Frank, M. Aribandi, S. Chopra, and K. N. Chintapalli, "Radiofrequency thermal ablation: computer analysis of the size of the thermal injury created by overlapping ablations," AJR Am J Roentgenol, vol. 177, pp. 777-82, Oct 2001.

[14] M. H. Chen, W. Yang, K. Yan, M. W. Zou, L. Solbiati, J. B. Liu, and Y. Dai, "Large liver tumors: protocol for radiofrequency ablation and its clinical application in 110 patients--mathematic model, overlapping mode, and electrode placement process," Radiology, vol. 232, pp. 260-71, Jul 2004.

[15] C. Baegert, C. Villard, P. Schreck, L. Soler, and A. Gangi, "Trajectory optimization for the planning of percutaneous radiofrequency ablation of hepatic tumors," Comput Aided Surg, vol. 12, pp. 82-90, Mar 2007.

[16] B. Davies, "A review of robotics in surgery," Proc Inst Mech Eng [H], vol. 214, pp. 129-40, 2000.

[17] M. H. David A. Simon, Takeo Kanade,, "Techniques for fast and accurate intrasurgical registration," Journal of Image Guided Surgery, vol. 1, pp. 17-29, 1995.

[18] N. Abolhassani, R. Patel, and M. Moallem, "Needle insertion into soft tissue: A survey," Medical Engineering \& Physics, vol. 29, pp. 413-431, 2007.

[19] A. M. Okamura, C. Simone, and M. D. O'Leary, "Force modeling for needle insertion into soft tissue," Biomedical Engineering, IEEE Transactions on, vol. 51, pp. 1707-1716, 2004.

[20] R. J. Webster, III, J. Memisevic, and A. M. Okamura, "Design Considerations for Robotic Needle Steering," in Robotics and Automation, 2005. ICRA 2005. Proceedings of the 2005 IEEE International Conference on, 2005, pp. 3588-3594.

[21] J. J. Craig, Introduction to Robotics Mechanics and Control Prentice Hall, 2005.

[22] S. P. DiMaio and S. E. Salcudean, "Interactive simulation of needle insertion models," IEEE Trans Biomed Eng, vol. 52, pp. 1167-79, Jul 2005.

[23] K. E. Chui C, Chen X, Hisada T, Sakuma I, "Combined compression and elongation experiments and non-linear modelling of liver tissue for surgical simulation " Medical and Biological Engineering and Computing, vol. 42, pp. 787-798, 2004. 


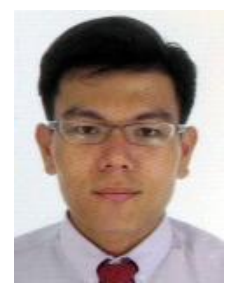

Liangjing Yang received his B.Eng. (Hons) degree from the Department of Mechanical Engineering, National University of Singapore, Singapore in 2008. $\mathrm{He}$ is currently a Research Engineer in the Department of Mechanical Engineering, National University of Singapore, Singapore. He is also a M.Eng. degree candidate there. His research interest lies in the area of augmented reality based surgery and medical robotics.

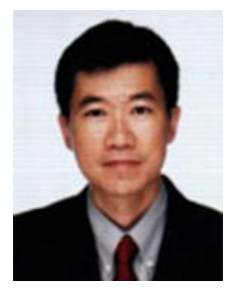

Chee-Kong Chui is currently an Assistant Professor in the Control and Mechatronics Group, Department of Mechanical Engineering, National University of Singapore, Singapore. He was the principal investigator of the Biomedical Simulation \& Device Design Project at the then Institute of Bioengineering prior to pursing a $\mathrm{PhD}$ in Biomedical Precision Engineering Lab, The University of Tokyo, Japan. His research interests include computer integrated and robot assisted surgery, human-machine interface, medical device design, biomechanical modeling and simulation.

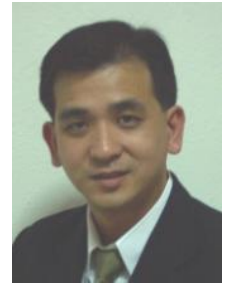

Stephen Chang is currently Consultant and Research Director of the Department of Surgery, National University Hospital, Singapore. He is a key developer of the laparoscopic approach to Hepatobiliary and Pancreatic surgery in this region and eagerly shares his skills with the other surgeons in Singapore. He has since published his experience in several major surgical journals. He is also active in developing Living Related Liver Transplant Program in Singapore and hopes to set up the programme to perform the donor operation laparoscopically. In addition to his surgical practice, Dr Chang has several research interests including pancreatic diseases, colorectal metastases, minimally invasive approach to biliary and stone diseases as well as robotic surgery. 